\title{
Giant Optical Transmission of a Subwavelength Slit Optimized Using the Magnetic Field Phase
}

\author{
O. T. A. Janssen and H.P. Urbach \\ Optics Research Group, Delft University of Technology, 2628CJ Delft, The Netherlands \\ G. W. 't Hooft \\ Philips Research Laboratories, 5656AA Eindhoven, The Netherlands
}

(Received 11 April 2007; published 25 July 2007)

\begin{abstract}
In this Letter, we show that the energy equivalent to that incident on a $4.7 \mu \mathrm{m}$ wide strip can be squeezed through a $50 \mathrm{~nm}$ wide slit in a metal film surrounded by grooves. This corresponds to a transmission efficiency of $9400 \%$, which can be even further enhanced by increasing the number of grooves. We use the phase of the magnetic field to explain that the ideal slit-to-groove distance is just over half the plasmon wavelength. In addition, we also optimize the groove depth and width. Such optimized transmission enhancement is very important for near-field devices.
\end{abstract}

After the discovery of surface-plasmon-enhanced transmission through a periodic configuration of holes in a metallic grating, there has been extensive research to explain this phenomenon and to optimize it [1]. Enhanced transmission through subwavelength holes has applications in high-resolution near-field microscopy and high-density optical storage. Since isolated subwavelength holes have low transmission efficiencies, surface-plasmon-enhanced transmission using a so-called bull's-eye pattern [2] can make a near-field application feasible.

The two-dimensional (2D) equivalent of the bull's-eye pattern in a metal film is shown in Fig. 1. It consists of a slit surrounded by several grooves. We shall use the coordinate system such that the grooves are periodic in the $x$ direction and the structure is independent of $y$. The role of the grooves is to transform transverse magnetic (TM) polarized incident light into surface plasmons that are partly transmitted through the slit.

It is possible to optimize this pattern by varying the groove period or pitch $a$ as is done in Ref. [3] as well as the groove depth $d$ as in Refs. [2,4]. However, the distance $b$ from the slit center to the nearest groove center has a more important influence on the transmission and has been investigated in the time domain using pulse illumination [5]. An optimal distance $b$ has been suggested based on interfering electric fields [6]. Further modifications of the original configuration have been studied. For example, grooves have been introduced on the exit surface to obtain more collimated beams of transmitted light [7-9]. The transmission enhancement of all suggested patterns is moderate, however. It was found in Refs. $[10,11]$ that the transmission does not increase further when there are more than 4 or 5 grooves on each side of the slit.

In this Letter, the remarkable efficiency of a slit surrounded by grooves is explained using the magnetic field component parallel to the slit. The interference of the magnetic field of the surface plasmons with the incident plane wave determines for which distance between the slit and the nearest groove the transmission becomes maximal. We therefore choose the distance $b$ such that constructive interference occurs at the slit entrance. We will also show that if the groove depth and width are carefully chosen, using more than 5 grooves on each side still improves the transmission.

In Ref. [6], the $E_{x}$ of the scattered field is found to be excited $\pi / 2$ out of phase with the $E_{x}$ of the incident field. This agrees with the phase $\pi$ found by us for the magnetic field [12]. Lezec and Thio proposed that the optimal distance is $b=0.75 a$, because then the $E_{x}$ field constructively interferes at the slit entrance with the incident field. However, since for a surface wave $E_{x}$ is small compared to $E_{z}$, maximizing the $H_{y}$ field gives much better results. Both the incident and the scattered fields have a strong $H_{y}$ component.

In this theoretical study, we simulate the slit surrounded by grooves using a 2D finite element method (FEM) [13]. The simulations are for a gold film in the $600-1000 \mathrm{~nm}$
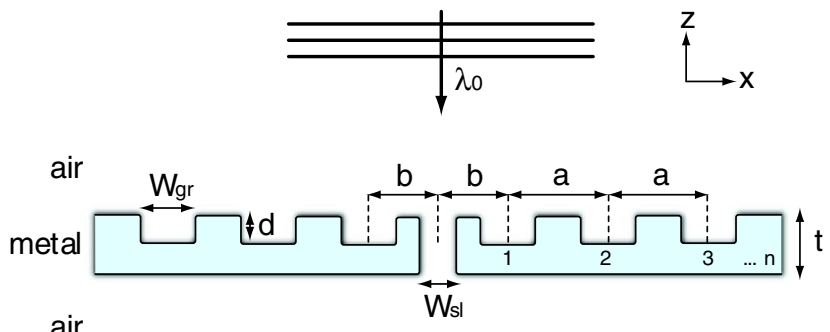

FIG. 1 (color online). Schematic representation of the 2D equivalent of the bull's-eye pattern used to optimize transmission through the slit. It consists of a metal film with thickness $t$, a slit with width $W_{\mathrm{sl}}$, and $n$ grooves on each side with width $W_{\mathrm{gr}}$ and depth $d$. The groove structure has period $a$ and slit-to-nearestgroove distance $b$. Above and below the film is air. A normal incident TM polarized plane wave with wavelength $\lambda_{0}$ and unit amplitude illuminates the pattern. In the original bull's-eye pattern, $a=b$. 
wavelength range, using realistic values for the permittivity of gold [14], so that absorption is taken into account. A film thickness $t=200 \mathrm{~nm}$ is chosen so that for incident light with $\lambda_{0}=800 \mathrm{~nm}$ there is a maximum for the FabryPérot resonance in the slit and consequently more transmission; see also [15].

The transmission efficiency of a slit surrounded by grooves is based on two phenomena, which we will discuss subsequently. The first is the collection of the incident light and its conversion into surface plasmons by the grooves. The second phenomenon is the interference of the surface plasmons with the incident light at the slit.

(i) Surface plasmon excitation using a periodic groove surface has been extensively studied [16]. For normal incident TM polarized light, the pitch or periodicity must be equal to the surface plasmon wavelength: $a=\lambda_{\text {sp }}$. We have optimized the duty cycle $W_{\mathrm{gr}} / a$, the groove depth $d$, and the number of grooves $n$ to maximize the energy propagated by the surface waves. A duty cycle of 0.5 for shallow grooves up to 0.6 for deeper grooves is most efficient for exciting plasmons, as is seen from Fig. 2. Although we use the plasmon field on a flat part of the metal film to determine the collected plasmon field amplitude, the field in the periodic groove structure will not be equal to that of a surface plasmon on a smooth surface. Of all possible modes in the periodic grooves, the dominant one experiences less metal than the smooth surface plasmon. This leads to a slightly lower wavelength of this mode than the surface plasmon wavelength [2] or a slightly higher optimal duty cycle for a pitch equal to that of the plasmon wavelength. In comparison, we found that a structure with metallic bumps instead of grooves is most efficient for a duty cycle between 0.4 and 0.5 .

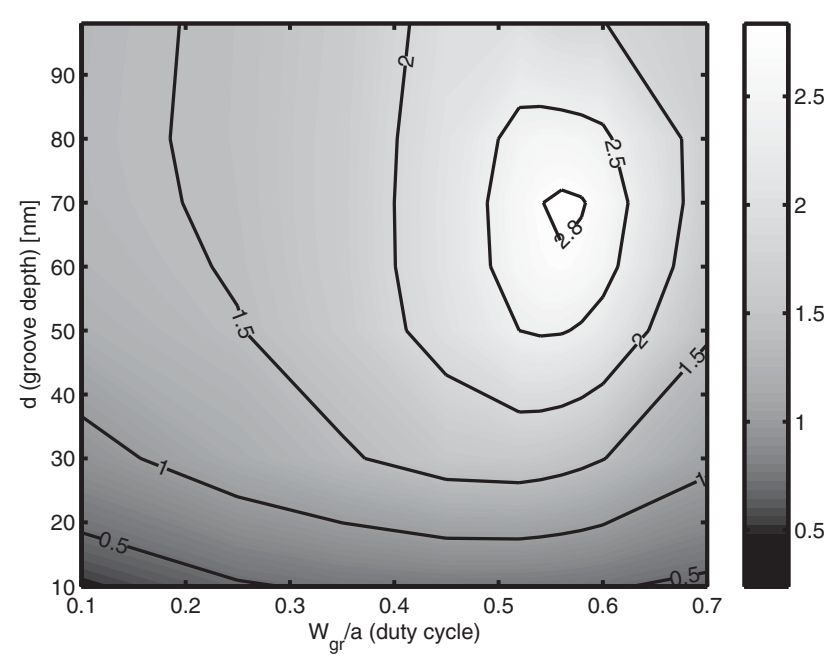

FIG. 2. Amplitude of the magnetic field of the surface plasmon on the upper metal surface at a distance of $7 \mu \mathrm{m}$ from the outer groove of a configuration with 11 grooves in a thick gold film, without a slit. The computations are for $\lambda_{0}=800 \mathrm{~nm}$, for which $a=\lambda_{\mathrm{sp}}=785 \mathrm{~nm}$.
Assuming an exponential decay, the energy radiated by a plasmon per period of the grating is approximately proportional to $4 \operatorname{Im}\left(k_{x}\right) a$. For $n$ periods, the energy radiated is $4 \operatorname{Im}\left(k_{x}\right) n a$. In the Born approximation, assuming a small groove depth $d$, the scattered field caused by a grating of $n$ periods is proportional to $n d$, and the scattered energy is thus proportional to $n^{2} d^{2}$. Combining these two relations, we find that, for a given number of grooves, there is an optimal groove depth $d_{\text {opt }} \propto n^{-(1 / 2)}$.

For gold we found, indeed, that, when more grooves are used, the optimal groove depth becomes smaller and scales with $n^{-1 / 2}$; see Fig. 3. Here we simulated a typical slit surrounded by grooves for which only the depth of the groove and the wavelength of the incident light is varied. Shallow grooves scatter surface plasmons less strongly into the far field, but they also excite surface plasmons less efficiently than deeper ones [17]. This is in agreement with the reciprocity principle [18]. For an optimal combination of groove depth and number of grooves, however, the simulations show that many shallow grooves collect more surface energy for transmission than fewer deeper ones. Absorption causes the much lower transmissions at wavelengths of $600 \mathrm{~nm}$ and less.

Consider next the excitation of a plasmon by only one groove. Using the FEM solution, we determine the phase of the magnetic $y$ component at a large distance from the groove. At such large distances, it may be assumed that the field is primarily due to the plasmon. If we assume that surface plasmons originate from the center of a groove, then we can deduce that the groove excites a plasmon roughly in antiphase with the local magnetic field of the incident light. To do so, we take the phase of the magnetic field calculated with the FEM at a sufficiently far distance

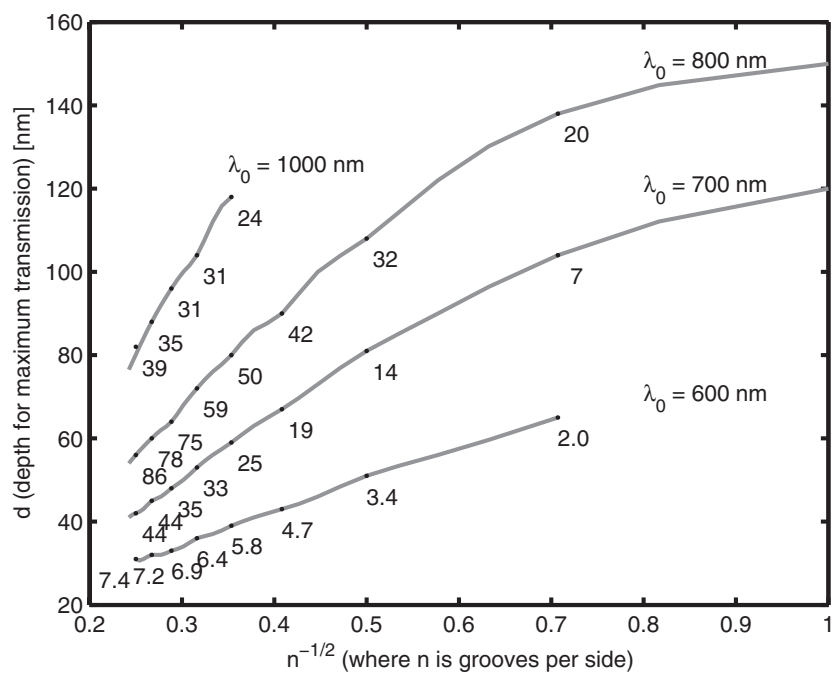

FIG. 3. Groove depth at which maximum transmission occurs for a single slit in gold surrounded by $n$ grooves. With $a=\lambda_{\mathrm{sp}}$, $W_{\mathrm{gr}} / a=0.56, b=0.55 a, W_{\mathrm{sl}}=50 \mathrm{~nm}$, and $t=200 \mathrm{~nm}$. The annotations give the total energy transmitted into the far field normalized by the energy incident on the slit opening. 


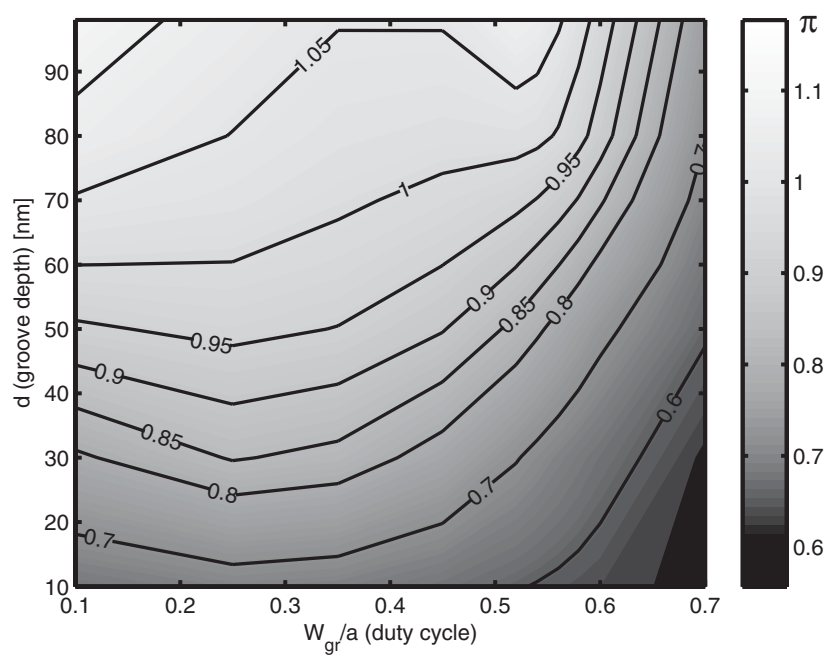

FIG. 4. Same as Fig. 2 but here the phase of the magnetic field of the excited plasmon in $\pi$ radians is plotted. The definition of this phase is given in the text.

of $7 \mu \mathrm{m}$ from the groove, where the field is dominated by the surface plasmon, but not so far that there is too little amplitude left. Using the plasmon wavelength, which is also determined from the field calculated with the FEM, we propagate this phase back to the groove center. The difference between this phase and the phase of the incident plane wave at the metal interface will be called the excitation phase of the surface plasmon field. This phase is found to be close to $\pi$ as is seen in Fig. 4. This holds to a large extent independently from the width of the groove. Because of other contributions to the scattered field than the surface plasmon, the surface plasmon excitation phase is different from the phase of the local total scattered magnetic field at the groove centers. More results about this plasmon excitation phase can be found in Ref. [12].

(ii) The distance $b$ from the slit to the nearest groove is very important for the transmission. A large plasmon field on the metal surface due to an optimized groove pattern does not automatically result in large transmission through the slit. In the original proposal of the bull's-eye pattern [2], the slit-to-groove distance was identical to the pitch of the grooves, so $a=b$. Because the pitch $a$ is equal to the plasmon wavelength, the plasmon fields generated at the grooves all constructively interfere. But, since the phase of the magnetic field of the surface plasmon wave is in antiphase with the incident magnetic field at the center of a groove, it is for this value of $b$ also in antiphase with the incident magnetic field at the center of the slit. This results in a low transmission through the slit as is seen in Fig. 5.

When the distance between the slit and the nearest groove is half the surface plasmon wavelength, the magnetic field of the plasmon is in phase with the incident field at the slit. The constructive interference results in an increased magnetic field intensity at the slit entrance, which causes a strong transmission enhancement, as seen in the bottom of Fig. 5. We used narrow grooves for these
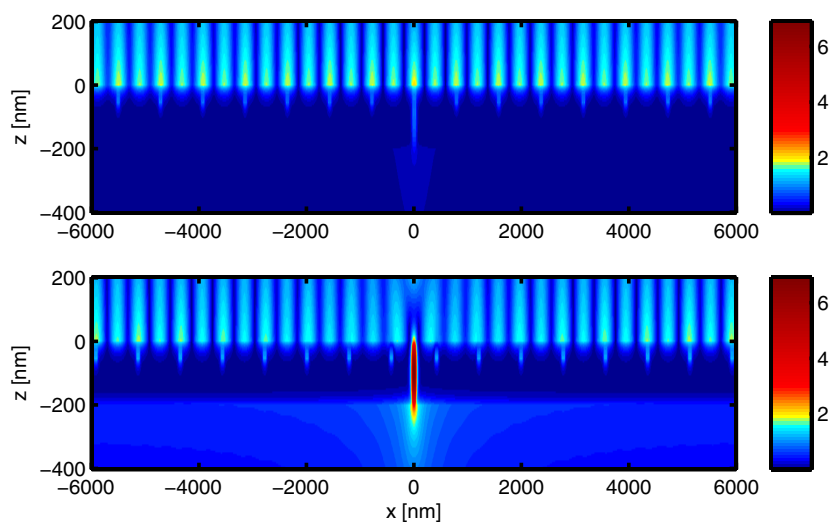

FIG. 5 (color online). Scattered magnetic field amplitude near two structures with $\lambda_{0}=800 \mathrm{~nm}, W_{\mathrm{sl}}=50 \mathrm{~nm}, W_{\mathrm{gr}}=50 \mathrm{~nm}$, $a=\lambda_{\mathrm{sp}}=785 \mathrm{~nm}, d=56 \mathrm{~nm}, t=200 \mathrm{~nm}$, and $n=33$. In the upper figure, $b=a$, and, as is seen, there is destructive interference at the slit entrance which results in a low transmission. In the lower figure, $b=0.55 a$, for which there is constructive interference which results in high transmission. The scattered field is defined as the total field minus the field of the analytical solution to the air-gold-air multilayer, i.e., the additional field caused by the grooves and slit only.

simulations $W_{\mathrm{gr}}=50 \mathrm{~nm}$, because they are more sensitive to the interference pattern so that the difference between destructive and constructive interference is more clearly visible.

An actual phase of $0.93 \pi$ is found in Fig. 4, for an optical periodic groove structure with a groove depth of $70 \mathrm{~nm}$ and a duty cycle of 0.56 . We therefore expect constructive interference and maximum transmission of the slit with the same groove structure at a distance that is $7 \%$ larger than half the plasmon wavelength [12], hence $b \approx 0.54 a$. This agrees well with the computed results illustrated in Fig. 6. The simulated transmission maxima for a groove depth of 20 and $40 \mathrm{~nm}$ are also plotted in this figure. Note that the prediction is not exact, since it is based on a nonrigorous intuitive model in which the surface plasmon is considered to be the dominant feature and other surface wave contributions are neglected.

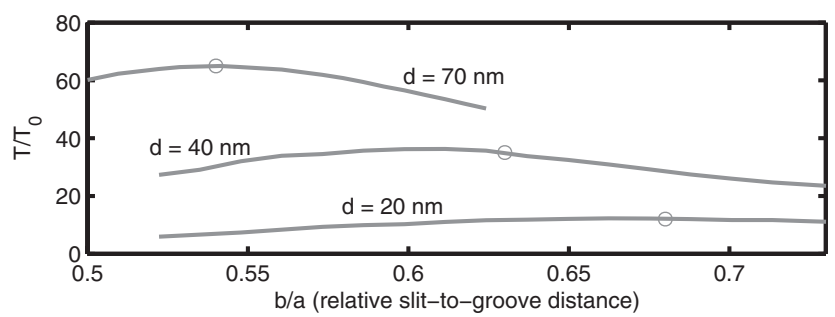

FIG. 6. Total energy transmitted into the far field $T$ normalized to the energy incident on the slit opening $T_{0}$. The parameters of the configuration are $\lambda_{0}=800 \mathrm{~nm}, \quad a=\lambda_{\mathrm{sp}}=785 \mathrm{~nm}$, $W_{\mathrm{gr}} / a=0.56, t=200 \mathrm{~nm}, W_{\mathrm{sl}}=50 \mathrm{~nm}$, and $n=11$. The transmission is given for structures with three different groove depths $d$. The circles indicate the predicted transmission maxima based on the phase shown in Fig. 4. 


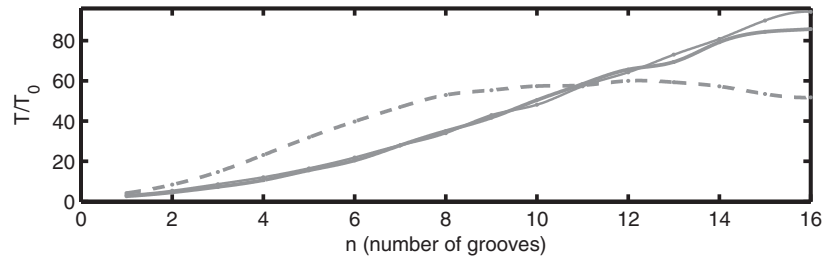

FIG. 7. The normalized transmission enhancement as a function of the number of grooves on either side of the slit for a pattern with optimized groove depth $d=56 \mathrm{~nm}$ (thick solid line) and for a pattern with nonoptimal groove depth $d=$ $80 \mathrm{~nm}$ (dashed line). The pattern is simulated with $\lambda_{0}=$ $800 \mathrm{~nm}, a=\lambda_{\mathrm{sp}}=785 \mathrm{~nm}, W_{\mathrm{gr}} / a=0.56, b=0.55 a$, and $t=$ $200 \mathrm{~nm}$. The thin solid line is the transmission for a pattern identical to the thick solid line with a slightly smaller groove period $a=779 \mathrm{~nm}$.

In addition, we found that if the groove depth is not optimal, the transmission enhancement saturates for relatively small values of $n$. With the groove depth estimated from the data shown in Fig. 3, we can use 16 grooves on each side and still obtain enhancement, as is seen in Fig. 7. As previously noted, the surface mode in the periodic groove structure will have a slightly lower wavelength than that of the theoretical surface plasmon. By fine-tuning the groove period $a$ by only $1 \%$, we can slightly improve the enhancement if we choose $a=779 \mathrm{~nm}$. Figure 8 shows that, for $\lambda=800 \mathrm{~nm}$ and $n=11$, the total energy squeezed through the slit is the same as the energy incident on a 3.2-4 $\mu \mathrm{m}$ wide strip. This corresponds to $20 \%$ of the width of the entire pattern and holds for a large range of slit widths. With $n=16$, we can even squeeze the energy incident on a $4.7 \mu \mathrm{m}$ wide strip through a $50 \mathrm{~nm}$ wide slit, corresponding to a transmission efficiency of $9400 \%$. Figure 7 shows that the limit is not reached and even higher transmission can be expected. Eventually, very shallow grooves are required, and the length of the groove pattern will approach the damping length of a smooth surface

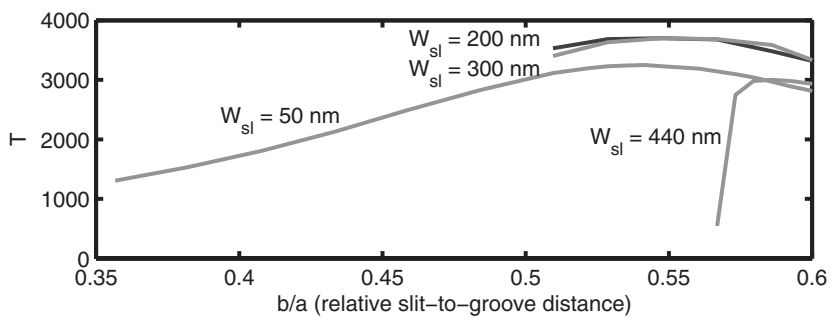

FIG. 8. Total energy transmitted into the far field $T$ normalized to the energy incident per unit length. Note that, unlike in the other figures, the transmission here is not normalized on the slit width. The configuration has parameters $\lambda_{0}=800 \mathrm{~nm}, a=$ $\lambda_{\mathrm{sp}}=785 \mathrm{~nm}, W_{\mathrm{gr}} / a=0.56, d=70 \mathrm{~nm}, t=200 \mathrm{~nm}$, and $n=11$. The curves cut off at a certain value of $b / a$, where the slit touches the nearest groove. plasmon. By using a similar groove corrugation on the exit surface, even more of the transmitted energy of the slit ends up in the far-field transmission. Obtaining such enhancements in a laboratory will be a challenge for experimental physicists.

In conclusion, we have shown that enhanced transmission can be explained and optimized by considering the condition for constructive interference between the magnetic field of the incident and the scattered field at the slit entrance. We optimized the groove depth, groove width, number of grooves, and slit-to-groove distance to improve the transmission of the slit. Based on studies in which 2D slit-groove and 3D slit-hole interaction are compared $[19,20]$, we expect that the optimal slit-groove distance we found also holds for 3D bull's-eye patterns.

[1] C. Genet and T. W. Ebbesen, Nature (London) 445, 39 (2007).

[2] T. Thio, K. M. Pellerin, R. A. Linke, H. J. Lezec, and T. W. Ebbesen, Opt. Lett. 26, 1972 (2001).

[3] T. Thio, H. J. Lezec, T. W. Ebbesen, K. M. Pellerin, G. D. Lewen, A. Nahata, and R. A. Linke, Nanotechnology 13, 429 (2002).

[4] A. Degiron and T. Ebbesen, Opt. Express 12, 3694 (2004).

[5] A. Agrawal, H. Cao, and A. Nahata, Opt. Express 13, 3535 (2005).

[6] H. J. Lezec and T. Thio, Opt. Express 12, 3629 (2004).

[7] F. I. Baida, D. Van Labeke, and B. Guizal, Appl. Opt. 42, 6811 (2003).

[8] L. Martín-Moreno, F. J. García-Vidal, H. J. Lezec, A. Degiron, and T. W. Ebbesen, Phys. Rev. Lett. 90, 167401 (2003).

[9] L. B. Yu, D. Z. Lin, Y. C. Chen, Y. C. Chang, K. T. Huang, J. W. Liaw, J. T. Yeh, J. M. Liu, C. S. Yeh, and C. K. Lee, Phys. Rev. B 71, 041405(R) (2005).

[10] F. J. García-Vidal, H. J. Lezec, T. W. Ebbesen, and L. Martín-Moreno, Phys. Rev. Lett. 90, 213901 (2003).

[11] T. Ishi, J. Fujikata, and K. Ohashi, Jpn. J. Appl. Phys. 44, L170 (2005).

[12] O. T. A. Janssen, H. P. Urbach, and G. W. 't Hooft, Opt. Express 14, 11823 (2006).

[13] X. Wei, H. P. Urbach, and A. J. Wachters, J. Opt. Soc. Am. A 24, 866 (2007).

[14] Handbook of Optical Constants of Solids, edited by E. D. Palik (Academic, New York, 1985), ISBN 0-125-44420-6.

[15] Y. Xie, A. Zakharian, J. Moloney, and M. Mansuripur, Opt. Express 13, 4485 (2005).

[16] H. Raether, Surface Plasmons on Smooth and Rough Surfaces and on Gratings (Springer-Verlag, Berlin, 1988), ISBN 3-540-17363-3.

[17] J. A. Sánchez-Gil, Phys. Rev. B 53, 10317 (1996).

[18] Electrodynamics of Continuous Media, edited by L.D. Landau and E. M. Lifschitz (Addison-Wesley, Reading, MA, 1960), ISBN 0-080-09105-9.

[19] P. Lalanne and J. P. Hugonin, Nature Phys. 2, 551 (2006).

[20] G. Gay, O. Alloschery, B. V. de Lesegno, C. O’Dwyer, J. Weiner, and H. J. Lezec, Nature Phys. 2, 262 (2006). 